\title{
Detecção de falhas em couro caprino wet blue a partir da utilização de técnicas de Visão Computacional e Inteligência Artificial
}

\author{
Carlos Estevão Bastos Sousa ${ }^{1}$, Cláudio Marques de Sá Medeiros ${ }^{1}$, \\ Renato Francisco Pereira ${ }^{1}$, Alcides Andrade Neto ${ }^{1}$ \\ ${ }^{1}$ Instituto Federal de Educação Ciência e Tecnologia do Ceará (IFCE), \\ Av. Treze de Maio, 2081, Benfica, 95, Fortaleza, Ceará, 60040-215, Brasil \\ \{carlosestevaobs, claudiosa1965, renato.francisco.pereira\} @gmail.com, \\ alcides.andradedifce.edu.br
}

\begin{abstract}
Traditional tanneries acquire hides, in most cases, from small rural producers. Due to the rustic creation format, they are received with different types of defects. Such skins go through several processes until they are called wet blue. At this stage of production, qualification is carried out, which is based on the number of faults in the leather piece to define its level of quality. The nodetection of the failures can cause several financier losses to the sector. However, despite the difficulties encountered, the growth of this type of industry becomes clear. Thus, a methodology is proposed which presents an accuracy of $96.31 \%$ in the detection of flaws in leather parts wet blue.
\end{abstract}

Resumo. Os curtumes tradicionais adquirem peles, na maioria dos casos, de pequenos produtores rurais. Devido ao formato rústico de criação, elas são recebidas com diversos tipos de defeitos. Tais peles, passam por diversos processos até serem denominadas como wet blue. Nesse estágio de produção, é realizada a qualificação, que se baseia na quantidade de falhas existente na peça de couro para definir o seu nível de qualidade. A não detecção de falhas pode acarretar diversos prejuízos ao setor. No entanto, apesar das dificuldades encontradas, torna-se nítido o crescimento desse tipo de indústria. Dessa forma, é proposta uma metodologia a qual apresenta uma acurácia de 96,31 \% na detecção de falhas em peças de couro wet blue.

\section{Introdução}

A produção de couros vem aumentado mundialmente, ao mesmo tempo que vem se verificando um deslocamento da base de produção dos países desenvolvidos para os países em desenvolvimento. Logo, deve-se a uma combinação de fatores relacionados com a disponibilidade de matéria prima, mercado, custos, entre outros [Gutterres 2003].

No último senso brasileiro (2017), foram catalogados na região nordeste do país aproximadamente 7.660.173 caprinos [Embrapa 2018]. No entanto, apesar do quantitativo existente, entre outros fatores, a baixa qualidade das peles produzidas é um fator de grande importância para a redução da produção de couro nessa região.

Para que as peles cheguem ao consumidor final, elas devem ser submetidas a diversas etapas que perfazem desde a criação do animal, abate, extração da pele e ao pré- 
processamento. Neste último, ao qual passa a ser denominada como wet blue é qualificada entre 7 categorias, sendo a primeira, a melhor e a última, a de pior qualidade.

Conforme o trabalho proposto por [Renato Pereira 2018], o índice de concordância entre dois especialistas em qualificação de couro, que trabalham em uma mesma empresa, é de apenas 41,37\%. Dessa forma, torna-se nítida a problemática no processo de definir o nível de qualidade para as peças de couro.

Diante do exposto, os autores propõem a elaboração de um sistema computacional utilizando técnicas de Visão Computacional e Inteligência Artificial para a detecção de falhas em couro caprino, com o intuito de auxiliar os profissionais dos curtumes. A expectativa é que a utilização de tal ferramenta possa contribuir com o setor têxtil e assim, garantir uma maior confiabilidade na detecção de falhas, procedimento primordial para a identificação do nível de qualidade das peças de couro.

O presente artigo ainda possui 7 Seções. Na Seção 2 são apresentados os trabalhos relacionados a temática em questão. Nas Seções 3 e 4 são apresentados, respectivamente, o processo de aquisição do conjunto de imagens e as técnicas de extração de atributos utilizadas. A parametrização dos classificadores é apresentada na Seção 5. Na Seção 6 é apresentado o processo de detecção de falhas em amostras de couro caprino. Na Seção 7, são apresentados os resultados obtidos e, por fim, na Seção 8, são apresentadas as considerações finais.

\section{Trabalhos relacionados}

[Amorim et al. 2010] propõem um trabalho de classificação de falhas em peças de couro de cabra em dois tipos de imagens: couro cru e wet blue. Para a aquisição de atributos são utilizados Gray-Level Co-Occurrence Matrix (GLCM), filtros Gabor e dois espaços de cores. A partir dos atributos adquiridos são realizados 3 experimentos. $\mathrm{O}$ primeiro consiste em classificar os dados brutos, sem pré-processamento dos mesmos, através dos classificadores K-Nearest Neighbors (KNN), Bayes e Support Vector Machine (SVM), no qual, destaca-se a aplicação do classificador KNN, com taxa de precisão média de $95,9 \%$ para peles de couro cru e $93,8 \%$, para wet blue. No segundo experimento são utilizadas técnicas de redução de atributos como FisherFace, Chen Linear Discriminant Analysis (CLDA), Direct LDA (DLDA), Yang LDA (YLDA) e Kernel LDA (KLDA) e, em seguida, os dados resultantes são novamente classificados com os mesmos classificadores do primeiro experimento. Nesta etapa, a melhor combinação surge através do CLDA e KNN, obtendo uma taxa de precisão média de 92,2 \% para peças de couro cru e 90,3 $\%$, para wet blue. O terceiro experimento consiste em detectar as falhas, informar a sua localização e comparar com as informadas pelo profissional especialista, esta etapa não demonstrou resultados satisfatórios devido ao número de amostras utilizadas pelo autor.

[Pereira et al. 2018], propõe a detecção de falhas com base na criação de janelas de tamanho quadrado (fragmentos de imagens). Desta forma, cada imagem de couro caprino é fragmentada em tamanhos de 51, 101, 151, 201, 251 e 301 pixels. Em cada janela obtida aplica-se os métodos de extração de atributos Gray-Level Co-Occurrence Matrix (GLCM), Local Binary Pattern (LBP), Momentos e Structural Co-Occurrence Matrix (SCM). Com os dados obtidos, provindos das janelas elaboradas, são implementadas as classificações de falhas, a partir dos classificadores K-Nearest Neighbors (KNN), Bayes, Multilayer Perceptron (MLP) e Support Vector Machine (SVM). Isto posto, a melhor acurácia surge a 
partir de janelas de 51 x 51 pixels, extrator LBP e classificador MLP, com acurácia média de 90,25\%. Quanto à qualificação das peles, dentre 7 categorias, o autor adquire atributos, a partir de regiões detectadas isentas de falhas e, a partir do classificador SVM, obtém uma acurácia média de $86,86 \%$.

\section{Aquisição dos conjuntos de imagens de amostras de falhas em couro caprino}

Para a aquisição dos conjuntos de imagens de couro caprino, os autores fizeram uso de uma estrutura desenvolvida por [Santos Filho 2013], ela possui iluminação própria composta por duas lâmpadas fluorescentes de $40 \mathrm{~W}$ dispostas de modo a não gerar sombras, um suporte para câmera situado a 1,5 m acima da superfície da mesa, a qual possui fundo preto com o intuito de facilitar a extração do plano de fundo do objeto de interesse.

Quanto ao processo de captura e digitalização das imagens, é utilizada uma câmera Canon modelo T3 Rebel de 12.2 MP, zoom óptico de 55x e lente EF-S 18-55mm. Nesta etapa são adquiridos 312 registros fotográficos de couro caprino, distribuídos por classe, conforme apresentadas na Tabela 1.

Tabela 1. Quantidade de peles recebidas.

\begin{tabular}{c|ccccccc}
\hline Qualidade & 1 & 2 & 3 & 4 & 5 & 6 & 7 \\
\hline Quantidade & 21 & 44 & 48 & 50 & 49 & 50 & 50 \\
\hline
\end{tabular}

A aquisição do conjunto de amostras de regiões boas e regiões com falhas é executada com o auxílio de profissionais do curtume. Ela consiste em abrir imagens em um software editor, mostrá-la para um especialista, no qual ele aponta a uma determinada área. Após este processo, as coordenadas $X$ e $Y$ do local detectado e demais atributos que referenciam a amostra de couro, como por exemplo a sua denominação (normal, sinais leves, sarna, etc) e o nome do amostra de couro caprino, são salvas em um arquivo, criando assim, um relatório de localizações. Este é distribuído conforme apresentado na Tabela 2.

Tabela 2. Distribuição do conjunto de imagens elaborado relacionado a falhas em couro caprino.

\begin{tabular}{c|l|c}
\hline Rótulo & Região detectada & Número de amostras \\
\hline 0 & Normal (sem falhas) & 1669 \\
1 & Sinais leves ou picadas de mosquito & 477 \\
2 & Sinais mais graves ou buracos & 97 \\
3 & Sarna & 93 \\
4 & Cortes de faca leve & 462 \\
5 & Riscos de arame & 168 \\
6 & Marcas de espinhos ou demais cicatrizes da vegetação & 253 \\
7 & Bexigas & 20 \\
8 & Cicatrizes & 46 \\
9 & Gordura vegetal & 252 \\
10 & Má conservação & 317 \\
11 & Conservação forte & 65 \\
\hline
\end{tabular}

Conforme apresentado, com a metodologia citada, é elaborado um conjunto de imagens ao qual possui 3919 amostras. Salienta-se que os tipos de falhas detectados nesta, são organizados conforme apresentados pelos especialistas em qualificação de peças de couro. 


\section{Formação dos conjuntos de dados}

A etapa de extração de atributos para a geração dos conjuntos de dados é realizada a partir dos métodos Local Binary Pattern - LBP [Ojala et al. 2002] e Gray Level Cooccurrence Matrix - GLCM [Haralick et al. 1973].

[Huang et al. 2011] afirma que nos últimos anos, o LBP despertou crescente interesse no processamento de imagens e Visão Computacional. [Mohanaiah et al. 2013] cita que uma das propriedades mais importantes deste operador é a simplicidade computacional, ela torna possível analisar imagens em configurações desafiadoras em tempo real.

Quanto ao extrator GLCM, seu objetivo é descrever textura através de um conjunto de características para as ocorrências de cada nível de cinza nos pixels da imagem considerando múltiplas direções [Schwartz and Pedrini 2003]. Dessa forma, o tamanho da matriz é determinado a partir da quantidade distinta de níveis de pixels contidos na imagem original [Annadurai 2007]. [Haralick et al. 1973] propôs um método ao qual pode-se obter 14 medidas estatísticas de textura a partir do uso dela, no entanto, apenas 13 são de fato utilizadas, pois a última apresenta instabilidade computacional.

Para o processo de extração de atributos faz-se necessário a elaboração do tamanho dos fragmentos a serem utilizados para a aplicação de tais métodos. Desta maneira, dadas as coordenadas $X$ e $Y$ referente ao pixel central da região destacada pelo especialista, citada anteriormente, considera-se uma área de tamanho $\frac{T}{2}$ acrescentado à direita, esquerda, superior e inferior ao mesmo (Figura 1).

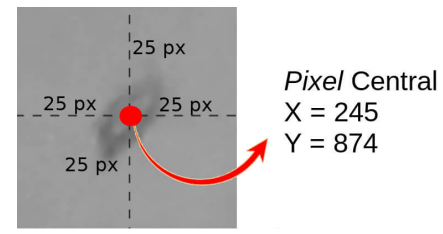

Figura 1. Exemplo de área a ser extraída as características com valor $\mathbf{T}=50$.

Ao analisar a Figura 1, nota-se que resultado obtido é uma área de tamanho $T+1$ pixels, no qual o valor 1 refere-se ao pixel central. Nesse sentido, são elaborados fragmentos com base nos valores para $T$ iguais a 10, 20, 30, 40, 50, 75 e 100, o que proporcionam a elaboração de diversos conjuntos de dados.

\section{Parametrização dos classificadores}

Os autores fazem uso de 7 classificadores para a identificação de falhas em couro caprino: Perceptron, Regressão Logística, K-nearest neighbor (KNN), Classificador de Fischer (baseado na regra de Bayes), Nä̈ve Bayes, Multilayer Perceptron (MLP) e Support Vector Machine (SVM).

Para o classificador perceptron simples de única camada, com o intuito de verificar a linearidade ou não dos conjuntos de dados, utiliza-se as taxas de aprendizado com os seguintes valores: $0.01,0.05,0.1,0.5$, função sinal e redução linear ou exponencial da taxa de aprendizado.

Para o algoritmo, é condicionado para o término do treinamento a taxa de erro de 
$10^{-5}$ e são implementadas 2000 iterações, as quais, os pesos que obtiverem uma maior acurácia são escolhidos como os melhores e representam o resultado do treinamento.

Quanto ao uso do algoritmo Logistic Regression, semelhante ao algoritmo anteriormente citado, é condicionada para o término do treinamento a taxa de erro de $10^{-5}$ e/ou 2000 iterações. O valor de C, ou seja, o parâmetro de penalidade é inserido a partir dos seguintes valores $0.01,0.1$ e 1.0 .

O KNN não necessita de treinamento, desta forma, são escolhidos apenas os valores para $K$, que são: 3, 5, 7, 9 e 11 e implementado o método de mensuração através da distância Euclidiana entre as amostras.

Quanto ao classificador Nä̈ve Bayes é aplicada o tipo de distribuição Gaussiana.

Para o classificador MLP são implementadas diversas taxas de aprendizado, que variam entre 0.1 a 0.5 , redução linear ou exponencial dessas taxas, funções de ativação Logística ou Tangente Hiperbólica, diversos números de neurônios na camada oculta inseridos empiricamente com valores entre 2 e 100 neurônios.

O encerramento do treino do MLP é condicionado a uma taxa de erro de $10^{-5}$ ou o máximo 2000 épocas, no qual, os melhores pesos obtidos em cada, representam o resultado do treinamento do modelo.

Com relação ao classificador SVM, são utilizados 4 tipos de kernel; linear, RBF, polinomial e sigmoide. $\mathrm{O}$ valor de $\mathrm{C}$, ou seja, o máximo de 500 iterações e o parâmetro de penalidade é inserido a partir dos seguintes valores $0.01,0.1,1.0$ e 10 .

É válido ressaltar que para todos os classificadores é utilizada a técnica de validação cruzada $K$-Fold, com valores para $K$ iguais a 10. A aplicação deste método consiste em dividir o conjunto total de dados do treinamento em $K$ subconjuntos de mesmo tamanho. Assim, cada subconjunto é utilizado para testes, enquanto que o restante do conjunto é aplicado para estimação dos parâmetros.

\section{Detecção de falhas em couro caprino}

Os autores iniciaram o processo de classificação considerando todos os tipos de falhas, de forma individual. Assim, foi executado um processo de classificação multi-classe, ou seja, baseada no uso de 12 classes (vide Tabela 2). Neste processo obteve-se como melhor modelo a utilização de fragmentos de 75 pixels, extrator GLCM e classificador $\mathrm{SVM}_{\text {Linear }}$, nos quais, conforme apresentado na Tabela 3, demonstram diversos erros de classificação.

No processo de classificação citado, e apresentado na Tabela 3, nota-se a ocorrência de muita confusão entre todas as subclasses de falhas. Desse modo, levando em consideração que para qualificar peças de couro caprino, não há a necessidade distinguir os tipos de falha, mas apenas fazer a sua detecção, os autores propõem a transformação de tal problema em uma classificação binária, ou seja, a partir da designação de regiões normais ou de falha.

Na nova metodologia para o treinamento o conjunto de dados é dividido em 2670 amostras divididas igualmente entre normais e falha para treino/teste. Todo o restante do universo de dados, 334 amostras normais e 915 falhas, é utilizado para validação. Logo, os dados utilizados no processo de aprendizagem são balanceados, o que garante melhores 
Tabela 3. Matriz de confusão obtida durante o processo de classificação entre diversos tipos de falhas e regiões normais

\begin{tabular}{c|cccccccccccc}
\hline Real \Predito & 0 & 1 & 2 & 3 & 4 & 5 & 6 & 7 & 8 & 9 & 10 & 11 \\
\hline 0 & 1384 & 111 & 0 & 0 & 110 & 0 & 2 & 27 & 32 & 1 & 0 & 2 \\
1 & 64 & 211 & 0 & 14 & 54 & 51 & 5 & 17 & 29 & 22 & 1 & 9 \\
2 & 0 & 1 & 88 & 3 & 1 & 2 & 1 & 1 & 0 & 0 & 0 & 0 \\
3 & 0 & 1 & 0 & 50 & 7 & 3 & 0 & 8 & 3 & 1 & 11 & 9 \\
4 & 41 & 37 & 0 & 29 & 187 & 31 & 0 & 97 & 12 & 4 & 0 & 24 \\
5 & 2 & 16 & 0 & 24 & 29 & 59 & 2 & 15 & 1 & 5 & 2 & 13 \\
6 & 3 & 3 & 0 & 3 & 33 & 7 & 192 & 5 & 1 & 6 & 0 & 0 \\
7 & 0 & 2 & 0 & 1 & 3 & 4 & 0 & 8 & 1 & 0 & 0 & 1 \\
8 & 3 & 5 & 0 & 2 & 6 & 9 & 0 & 10 & 10 & 0 & 0 & 1 \\
9 & 6 & 3 & 0 & 9 & 4 & 12 & 0 & 5 & 5 & 183 & 7 & 18 \\
10 & 0 & 0 & 0 & 65 & 7 & 22 & 0 & 5 & 2 & 27 & 141 & 48 \\
11 & 1 & 0 & 2 & 9 & 0 & 7 & 0 & 10 & 6 & 1 & 4 & 25 \\
\hline
\end{tabular}

condições para que os algoritmos obtenham bons resultados.

Após os processos de treinamento dos classificadores, é iniciada a etapa de detecção de falhas nas amostras de couro caprino. Esta divide-se em pré-processamento das imagens adquiridas, análise de fragmentos, classificação dos mesmos e segmentação das regiões classificadas como falha.

A etapa de pré-processamento, consiste em adquirir a imagem original, transformála em tons de cinza e limiarizá-la de modo a deixá-la de forma binária, ou seja, em tons brancos e pretos. Após o processo de limiarização aplica-se a técnica de detecção de contornos. Com a obtenção deles, toda a área interna do contorno mais externo detectado, recebe a cor preta. Resultando assim, em um plano de fundo na cor branca e toda a peça de couro caprino na cor preta.

Em seguida, é iniciada a verificação e substituição em cada pixel. Este processo consiste em verificar todos os pixels da imagem e, caso possuam cor preta, deverão ser substituídos pela cor do pixel respectivo da imagem original. O resultado de tais ações é a obtenção do objeto de interesse (a peça de couro caprino) e o plano de fundo na cor branca.

Após realizados todos os procedimentos descritos anteriormente, a área do objeto de interesse é mapeada de forma a criar uma lista com todas as posições dos eixo $X$ e $Y$, de acordo com o tamanho de fragmento utilizado.

Com a imagem processada e mapeada, é iniciada a detecção de falhas com base nas posições contidas na lista anteriormente citada. Neste estágio é utilizada a técnica de processamento paralelo, no qual,cada fragmento da imagem é direcionado para um núcleo do processador.

Durante o processo de detecção de falhas, caso a área analisada possua algum pixel na cor branca a mesma não será analisada. Em caso contrário, se identificada como falha, receberá a cor vermelha ou, de maneira oposta, manterá a cor de origem da imagem original.

\section{Análise dos resultados}

Um dos métodos para analisar se as soluções propostas de fato generalizam bem o problema é testá-las de forma prática. Portanto, com o intuito de medir o grau de acerto na 
detecção de falhas, imagens são analisadas a olho nu e comparadas com as análises feitas pelos algoritmos propostos.

Com base no método apresentado, é analisado e obtido o melhor modelo computacional para a detecção de falhas. Salienta-se que este é executado 50 vezes com o intuito de garantir a melhor solução. A Tabela 4 ilustra tais valores.

\section{Tabela 4. Resultados relacionados ao melhor modelo computacional com base em 50 execuções.}

\begin{tabular}{c|c|c|c|c}
\hline \multirow{2}{*}{ Ordem } & \multirow{2}{*}{ Tam. Fragmento } & \multirow{2}{*}{ Tempo médio } & \multicolumn{2}{|c}{ Acurácia Média (\%)/Desvio Padrão $(\sigma)$} \\
\cline { 3 - 4 } & & & Treinamento & Validação \\
\hline $\mathrm{SVM}_{R B F}$ & 51 pixels & $\approx 11$ segundos & $96.59 \pm 0.17$ & $95.95 \pm 0.38$ \\
\hline
\end{tabular}

Analisando a Tabela 4, nota-se que o tempo médio para a execução do processo de detecção de falhas é de apenas 11 segundos. Um tempo relativamente bom ao se comparar o processo manual, descrito por [Pereira et al. 2018], que varia entre 10 e 30 segundos dependendo da peça de couro

A partir da utilização de fragmentos de 51 x 51 pixels, da GLCM, com extração de atributos com base no trabalho de [Haralick et al. 1973] e classificador $\mathrm{SVM}_{R B F}$, os autores obtiveram, como melhor classificação, o resultado descrito na matriz de confusão apresentada na Tabela 5.

Tabela 5. Matriz de confusão proveniente do treinamento e validação do classificador $\mathbf{S V M}_{R B F}$, atributos obtidos a partir do extrator GLCM e fragmentos de 51 pixels

\begin{tabular}{c|cc|cc}
\hline \multirow{2}{*}{ Real Predito } & \multicolumn{2}{|c|}{ Treinamento } & \multicolumn{2}{c}{ Validação } \\
& Normal & Falha & Normal & Falha \\
\hline Normal & 1304 & 31 & 329 & 5 \\
Falha & 63 & 1272 & 41 & 874 \\
\hline
\end{tabular}

Conforme os dados contidos na Tabela 5, nota-se que o melhor modelo proposto possui uma acurácia de $96,47 \%$ no processo de treinamento e 96,31 \% na validação. Apresentando um resultado superior ao obtido com o modelo multi-classe, descrito na Seção 6.

\section{Considerações finais}

Como visto, todo o pré-processamento realizado contribuiu significativamente para a resolução do problema em um tempo considerado aceitável, em comparação com a detecção de falhas efetuada nos curtumes tradicionais.

Considerando que um especialista em qualificação de peles necessita apenas detectar falhas, sem a necessariamente identificá-las. Os autores obtiveram, a partir da metodologia proposta uma taxa de acurácia de $96,47 \%$ no treinamento e $96,31 \%$ na validação, o que em termos práticos pode ocasionar em melhorias na qualificação de peles nos curtumes. 


\section{Referências}

Amorim, W. P., Pistori, H., Pereira, M. C., and Jacinto, M. A. C. (2010). Attributes reduction applied to leather defects classification. 23rd SIBGRAPI Conference on Graphics, Patterns and Images, pages 353-359.

Annadurai, S. (2007). Fundamentals of Digital Image Processing. Always learning. Pearson.

Embrapa (2018). Novo censo agropecuário mostra crescimento de efetivo de caprinos e ovinos no nordeste. https://www.embrapa.br/cim-inteligencia-e-mercado-decaprinos-e-ovinos/busca-de-noticias/-/noticia/36365362/novo-censo-agropecuariomostra-crescimento-de-efetivo-de-caprinos-e-ovinos-no-nordeste. Acesso em $28 \mathrm{dez}$. 2019.

Gutterres, M. (2003). Desenvolvimento Sustentável em Curtumes. XVI Encontro Nacional da ABQTIC, Porto Alegre - RS.

Haralick, R. M., Shanmugam, K., and Dinstein, I. (1973). Textural features for image classification. IEEE TRANSATIONS ON SYSTEMS, MAN AND CYBERNETICS, SMC3(6):610-621.

Huang, D., Shan, C., Ardabilian, M., Wang, Y., and Chen, L. (2011). Local binary patterns and its application to facial image analysis: A survey. IEEE Transactions on Systems, Man, and Cybernetics, Part C (Applications and Reviews), 41(6):765-781.

Mohanaiah, P., Sathyanarayana, P., and GuruKumar, L. (2013). Image texture feature extraction using glcm approach. International Journal of Scientific and Research Publications., 3:1-5.

Ojala, T., Pietikäinen, M., and Mäenpää, T. (2002). Multiresolution gray-scale and rotation invariant texture classification with local binary patterns. IEEE Trans. Pattern Anal. Mach. Intell., 24(7):971-987.

Pereira, R., Medeiros, C., and Filho, P. P. (2018). Goat leather quality classification using computer vision and machine learning. International Joint Conference on Neural Networks - IJCNN, pages 01-08.

Renato Pereira, F. (2018). Qualificação de amostras de couro caprino utilizando visão computacional e inteligência computacional. Master's thesis, Instituto Federal de Educação, Ciência e Tecnologia do Ceará (IFCE), Fortaleza, CE, Brazil.

Santos Filho, E. (2013). Um sistema de visão computacional para classificação de couro caprino. Master's thesis, Universidade Federal do Ceará (UFC), Fortaleza, CE, Brazil.

Schwartz, W. R. and Pedrini, H. (2003). Método para classificação de imagens baseada em matrizes de co-ocorrência utilizando características de textura. III Colóquio Brasileio de Ciências Geodésicas. 